Cinémas

Revue d'études cinématographiques

Journal of Film Studies

\title{
Saouter, Catherine (direction). Le Documentaire. Contestation et propagande. Montréal : XYZ éditeur, 1996, 161 p.
}

\section{Suzanne Langlois}

Volume 8, numéro 3, printemps 1998

Cinélekta 2

URI : https://id.erudit.org/iderudit/024763ar

DOI : https://doi.org/10.7202/024763ar

Aller au sommaire du numéro

Éditeur(s)

Cinémas

ISSN

1181-6945 (imprimé)

1705-6500 (numérique)

Découvrir la revue

Citer ce compte rendu

Langlois, S. (1998). Compte rendu de [Saouter, Catherine (direction). Le

Documentaire. Contestation et propagande. Montréal : XYZ éditeur, 1996, 161 p.]

Cinémas, 8(3), 161-168. https://doi.org/10.7202/024763ar d'utilisation que vous pouvez consulter en ligne.

https://apropos.erudit.org/fr/usagers/politique-dutilisation/ 
SAOUTER, Catherine (direction). Le Documentaire. Contestation et propagande. Montréal : XYZ éditeur, 1996, 161 p.

Ce livre rassemble les communications présentées lors du colloque "Contestation et propagande, pratiques du documentaire " qui s'est tenu en 1995 à l'Université du Québec à Montréal dans le cadre du Congrès des sociétés savantes. Il s'agissait, pendant cette année centenaire du cinéma, de souligner l'engagement du documentaire tout au long de ce siècle. Ce n'est pas un hasard si un tel livre est publié au Canada, car depuis longtemps, face au géant culturel américain, ce pays a fait siens des parcours cinématographiques différents, le cinéma documentaire, le film d'animation, le court métrage. Catherine Saouter a réuni, pour parler des pratiques du film documentaire à toutes les époques et sur tous les continents, des chercheurs universitaires et des professionnels du cinéma d'ici et d'Europe. Dans tous les ouvrages de cette nature, les textes sont grandement variés, et c'est tant mieux pour le lecteur qui y puisera abondante matière à réflexion, tant pour les idées que pour la méthodologie. En voici un aperçu.

Vincent Patigny, de l'Institut Émile Vandervelde de Belgique, nous sert une substantielle entrée en matière avec son article "Linterdit documentaire". Il place sa réflexion dans la perspective des menaces politique et culturelle que représentent les nouvelles structures mondiales du discours médiatique, qu'il considère menaçantes pour la démocratie. L'Europe, forte de ses traditions et de ses spécificités philosophiques et culturelles, doit réagir et devrait proposer un nouveau paradigme, celui de l'identité en construction. Le point d'ancrage et de départ de Vincent Patigny est la communauté française de Belgique. Puisque la Belgique peut déjà s'enorgueillir de pratiques documentaires et 
sociales qui s'inscrivent dans une perspective contestataire, elle peut éclairer l'objectif commun de "garantir l'exercice de la démocratie dans les sphères informationnelles, cinématographiques et audiovisuelles». Deux étapes doivent s'emboîter: le cinéma doit porter une critique radicale du système de production cinématographique dominant, et il doit rendre visible ce qui est trop souvent occulté, les réalités sociales, politiques et culturelles. Cette analyse des rapports de production apparaît de prime abord engluée dans un vocabulaire marxiste, mais il faut garder l'œil sur le projet global, empreint d'humanisme. Les pratiques documentaires doivent affirmer l'esprit de révolte, réaffirmer l'intérêt général. De là tout le potentiel de cet article, qui replace la problématique du cinéma, ou du moins d'un certain cinéma, dans un large projet politique et social qui nécessite la participation à part entière non seulement du documentariste, mais également du citoyen dans l'exercice de la démocratie.

Toutefois, la question des réseaux parallèles aurait pu être évaluée plus largement par Vincent Patigny. Le cinéma n'est pas seulement mécanisme de consensus social, il peut également rompre cet engrenage ${ }^{\prime}$ et des pratiques contestataires, ou simplement différentes, ont un impact sur la représentation et la réception des problèmes contemporains. Deux exemples peuvent illustrer ces parcours. En 1932, le cinéaste américain Leo Seltzer, membre de la Workers' Film and Photo League, tourne des images de la crise économique et de la détresse urbaine qu'elle entraîne aux États-Unis. Les actualités refusent de les montrer dans les salles de cinéma et Seltzer prend la route avec ses bobines et montre ses images partout où il peut, en circuit non commercial. Il s'agit d'un public restreint par rapport à celui des salles commerciales, mais ce sont les images de Seltzer qui survivent dans le temps et c'est à travers elles que nous percevons et comprenons aujourd'hui un large pan de l'histoire sociale américaine des années trente y compris l'échec des actualités cinématographiques à rendre compte de l'environnement réel. En France, en 1962, de jeunes réalisateurs publient un Manifeste pour un cinéma parallèle et se proposent d'aborder de front quelques sujets tabous, dont celui de l'Algérie. Ils comptent expressément sur les réseaux non commerciaux pour assu- 
mer la visibilité de leurs films, et la distribution parallèle est au point à ce moment. Certains observateurs verront dans cette prise de conscience un tournant et l'événement véritable de cette époque du cinéma français ${ }^{2}$.

Dans sa présentation de documentaires contestataires, Vincent Patigny commence par Las Hurdes (Terre sans pain) de Luis Buñuel (Espagne, 1933) et mentionne que depuis ce film, toute dénonciation d'une société, d'une réalité sociale, reposant sur les mêmes prémisses, les mêmes conventions, les mêmes mythes est devenue nécessité et fondement du documentaire social. Ce film, nous rappelle-t-il, fut frappé d'interdit par la République espagnole parce qu'il donnait à voir une mauvaise image du pays. Mais, même frappé d'interdit, Las Hurdes est très connu et son impact se fait sentir largement. Nous aimerions savoir comment et pour qui ce film a néanmoins circulé. Comme pour les images de Leo Seltzer, la démonstration/contestation de la réalité sociale par Buñuel est devenue une part essentielle de la connaissance de l'Espagne des années trente. Si l'interdit fait partie de l'histoire de ce film, ses modes de diffusion ultérieure également. Ce n'est pas seulement l'interdit, mais l'œuvre dans sa longue durée qui témoigne de sa force contestataire.

Un autre sujet, abordé en conclusion par Vincent Patigny, nécessite un commentaire. À propos du film documentaire Zone rouge (1990), une coproduction de cinq ateliers de production indépendants qui ont réalisé un manifeste audiovisuel sur les réalités sociales contemporaines du bassin industriel qui s'étend de Manchester à Milan, l'auteur indique que le film fut diffusé le $1^{\mathrm{er}}$ mai 1990 en Belgique et en Allemagne, et sur TV5 Europe, mais que l'introduction de Paul Meyer fut censurée par la ZDF pour avoir "confondu le capitalisme et le nazisme dans un même procès". Vincent Patigny poursuit :

L'esprit de progrès se trouve une fois de plus confronté aux forces qui le représentent aux seins (sic) des grandes institutions de pouvoir/savoir médiatiques de nos sociétés contemporaines.

Au travers de Zone rouge, à l'heure de la réunification allemande, l'interdit porte sur la mémoire de 
l'Holocauste, l'interprétation de ce crime contre l'humanité, la vérité de sa représentation. Si le monde semble dire que certaines vérités doivent être tues, c'est la déraison qui perdure au détriment de la liberté de penser, de filmer (p. 33).

J'invite le lecteur à réfléchir et à se demander de quel esprit de progrès il s'agit. Si la recherche de la vérité nourrit le projet du documentaire social, il faut s'interroger sur cette affirmation qui se veut péremptoire. Quelle interprétation de l'Holocauste est interdite? Quelle vérité? Celle qui voudrait que ce crime contre l'humanité soit l'étape ultime du capitalisme? Pire encore, que les réalités sociales contemporaines du bassin industriel européen puissent être mises en parallèle avec les conditions qui ont prévalu pour les Juifs en Allemagne nazie et en Europe occupée, et que leurs causes soient confondues? Si tel est le cas, ce raccourci capitalisme/nazisme/holocauste est indécent.

Il n'y a pas que ce premier article qui aborde la question des pratiques documentaires et Mireille La France a ciblé un exemple pertinent de cinéma de contestation politique avec Taire des hommes (1968), un court métrage sur l'émeute du 24 juin 1968 à Montréal, et qui témoigne de l'abus de pouvoir et de la brutalité des policiers. Elle en fait une excellente mise en contexte en l'insérant dans les débats idéologiques de l'époque au sujet du cinéma. Reviennent ainsi dans l'analyse la question des circuits commerciaux et parallèles, la question du langage cinématographique du cinéma de contestation politique, et celle du public de ces films. La prise en compte de ces trois éléments est essentielle.

Yves Laberge, dans son (trop) court article à propos du documentaire "The Journey" de Peter Watkins, pose également une question essentielle en se demandant comment un cinéaste peut répondre à la propagande. Il est exact que ce terme a acquis une connotation péjorative, surtout après que deux décennies d'analyses ont suivi la voie ouverte par Jacques Ellul dans les années 60 avec pour conséquence un élargissement du champ d'application de la propagande au point de rendre le concept inutilisable en science politique. Depuis la fin des années quatre-vingt, des chercheurs tâchent de le redéfinir. Mais, remercions Yves Laberge de nous mettre plutôt sur la piste d'un livre ancien, 
datant de 1939 et récemment réédité, qui présente un choix d'autres procédés cinématographiques afin d'éviter le recours à la propagande: la distanciation, l'analyse, la réflexion. Ce sont également les choix de Peter Watkins, qui utilise les images télévisuelles pour dénoncer les limites et les pièges de l'information télévisée.

Le choix de Catherine Saouter d'inviter des professionnels à nous faire part de leur expérience de la pratique documentaire était judicieux. Des cinéastes (Colin Low, Daniele Segre, Daniel Garabédian) et des monteurs (Werner Nold et Louise Surprenant de l'ONF) contribuent des pistes de réflexion à partir de l'intéricur de la profession. La démarche du cinéaste, l'équipement cinématographique, le tournage, la découverte, les rencontres, l'organisation des images et la volonté de faire sens, tous, ils abordent ces questions. Il faudrait sans doute joindre l'article de Charles Perraton à ce groupe, car il aborde le cinéma documentaire à partir de l'appareil de prise de vues. Sa contribution, "La caméra à l'épaule comme dispositif de vision dans le cinéma direct», s'attache plus particulièrement à l'œuvre de Michel Brault et à comment le cinéma direct tente d'intégrer le spectateur à l'expérience perceptive et à celle de l'interprétation des images. Charles Perraton n'hésite pas à mettre le rôle du spectateur en perspective historique et à expliquer comment ce rôle a changé au fil du temps, d'observateur de la camera oscura, à producteur actif de l'expérience optique au début du XIX siècle, pour finalement aboutir à une redéfinition des tâches liées à l'observation avec l'arrivée du cinématographe.

Abordons, en dernier lieu, deux autres articles substantiels: Catherine Saourer réfléchit sur les rapports qu'entretiennent l'État, les citoyerıs et les médias, à partir de films documentaires canadiens sur la Deuxième Guerre mondiale, et Michèle Lagny nous entretient du cinéma documentaire français et de la question coloniale durant la période 1945-1955. La tentation de mettre en parallèle ces deux articles l'emporte, malgré des sujets fort éloignés l'un de l'autre, à cause du défi méthodologique qu'ils posent alors que leurs auteures sont engagées dans de vastes recherches qui dépassent le cadre strict des communications qu'elles ont présentées lors de ce colloque. 
Puisqu'elle réfléchit sur les rapports entre l'État, les citoyens et les médias, Catherine Saouter considère que les célébrations commémorant la Seconde Guerre mondiale ont fourni une matière de choix pour observer les manifestations de la conscience collective. La guerre permet d'observer comment les groupes se donnent d'eux-mêmes des définitions plus visibles qu'à l'accoutumée et l'État canadien, poursuit-elle, s'est doté d'une institution, l'Office national du film, précisément pour élaborer et servir cette image. Elle a donc choisi quatre films documentaires sur la Seconde Guerre mondiale, produits en 1940, 1942, 1962 et 1992. C'est le contenu discursif qui l'intéresse davantage ici, et ces films sont pris tels quels, «comme les témoignages et variations du discours social cheminant pendant les cinquante dernières années d'une collectivité» (p. 144).

Catherine Saouter cherche des réponses à deux séries de questions pertinentes. La première triade se présente comme suit: qu'est-ce que le Québec? qu'est-ce que le Canada? qu'est-ce que l'Empire britannique? La seconde s'attache au soldat: pour qui intervient le soldat canadien? contre qui? contre quoi? Les quatre documentaires n'ont pas su répondre à la première série de questions et céla est démontré de façon convaincante. Toutefois, répondre à la seconde série de questions en affirmant que le Canada, dans le cas d'un conflit aussi évident que la Seconde Guerre mondiale, n'a pas su inscrire sa réponse, via ses films, dans la mémoire collective, s'avère être une généralisation abusive. Ici se fait jour un réel problème méthodologique car, telle qu'elle se présente, cette conclusion semble avoir dicté dès le départ le choix des documents pour qu'ils correspondent au contenu ébauché dans la problématique.

Une telle enquête dans les variations du discours, pour être véritablement éclairante, doit se fonder sur un corpus aussi large que possible et, pour le sujet qui nous intéresse, il y a eu de nombreux films. La liste Seconde Guerre mondiale du catalogue de l'ONF en contient des dizaines ${ }^{3}$. Le troisième documentaire choisi par Catherine Saouter est Profil du combattant (ONF, 1962), cinquième épisode de la série Canada en guerre qui compte 13 épisodes d'une demi-heure. Si le soldat canadien ne sait pas pour qui, ni contre quoi il se bat dans Profil du combat- 
tant, cette constatation peut-elle servir de conclusion pour les autres épisodes et, par extension, pour l'ensemble de la production de l'ONF sur la Seconde Guerre mondiale? À titre d'exemples, le troisième épisode, Tenir, présente le rôle du Canada dans la protection des convois maritimes de ravitaillement des Alliés; $D$ plus 333, le douzième épisode, porte sur l'année 1945, la capitulation de l'Allemagne et la découverte des camps de concentration. Lanalyse du discours et les commentaires pertinents de Catherine Saouter au sujet des quatre films choisis font regretter l'insuffisance du nombre de documents consultés.

Finalement, le projet de saisir ce "discours social cheminant pendant les 50 dernières années» comprend inévitablement un continuum qui ne devient compréhensible que par la rencontre de la sémiologie et de la sociologie avec l'histoire. C'est précisément ce qu'a fait Michèle Lagny et il est nécessaire de contraster ces démarches pour saisir ce que peut représenter un tel apport méthodologique d'une discipline voisine telle que l'histoire. On connaît la contribution exemplaire de Michèle Lagny sur l'utilisation de la méthode historique du cinéma ${ }^{4}$. Dans l'article qui nous intéresse ici, l'objectif est d'analyser la fonction du documentaire sur la question coloniale dans le violent débat qui marque la vie politique française pendant l'après-guerre. Nous n'aborderons pas ses conclusions, les lecteurs sont encouragés à aller y voir de plus près, mais signalons le corpus utilisé pour ses recherches, car c'est sa méthodologie qui attire immédiatement l'attention et rend ses conclusions pertinentes et convaincantes. Pour y parvenir, elle met en place une analyse quantitative puis qualitative de cette production assez importante puisqu'il y a plus de 450 films concernant les colonies françaises, et $60 \%$ de cette production a été localisée par le groupe d'étudiants-chercheurs travaillant à la confection d'un catalogue des films documentaires français produits pendant la décennie 1946-1955. Un large corpus permet de saisir les continuités et les ruptures, et de mettre en perspective les éléments de l'enquête, l'esprit qui a présidé à la réalisation de ces films, les contraintes auxquelles il a fallu faire face, les caractéristiques et l'évolution du thème.

En toute liberté, certaines démarches et affirmations appelaient réaction et discussion; et cette discussion reste d'ailleurs 
bien ouverte, car le lecteur aura compris la grande richesse thématique et méthodologique de Documentaire. Contestation et propagande. Plusieurs textes sont foisonnants et demandent une lecture attentive, mais qu'on se rassure, le public visé est un public général, invité à apprendre et à réfléchir sur de vastes questions. Cette rencontre entre chercheurs et professionnels enrichit certainement notre compréhension de tout ce qui touche au documentaire, de l'intérieur et de l'extérieur de la profession, en donnant même à voir le centre nerveux de nos perceptions.

Suzanne Langlois

McGill University

\section{NOTES}

1 Robert Sklar ct Charles Musser se sont intéressés aux mouvements oppositionnels qui utilisent le cinéma comme mécanisme de résistance. Voir l'ouvrage publié sous leur direction, Resisting Images (Philadelphie: Temple University Press, 1990).

2 Francis Courrade, Les Malédictions du cinéma français. Une histoire du cinéna françatis parlant (1928-1978) (Paris: Alain Moreau, 1978), p. 264.

3 LOffice national du film du Canada permet la consultation d'une grande partic de son catalogue sur son site internet: www.onf.ca

4 Michèle Lagny, De l'histoire du cinéma. Méthode historique et histoire du cinénu (Paris: Armand Colin, 1992). 